\title{
2D interfacial exchange-diffusion has the potential to augment spatiotemporal precision of $\mathrm{Ca}^{2+}$ signalling
}

\author{
Cornelis van Breemen ${ }^{1}$, Nicola Fameli ${ }^{1}$, and Klaus Groschner ${ }^{2}$ \\ ${ }^{1}$ University of British Columbia, Vancouver, BC, Canada \\ ${ }^{2}$ Gottfried Schatz Research Center, Medical University of Graz, Graz, Austria
}

November 1, 2021

\begin{abstract}
Nano-junctions between the endoplasmic reticulum and the cytoplasmic surfaces of the plasma membrane and other organelles shape the spatiotemporal features of biological $\mathrm{Ca}^{2+}$ signals. Herein we propose that 2D $\mathrm{Ca}^{2+}$ exchange-diffusion on the negatively charged phospholipid surface lining the nano-junctions participates in guiding $\mathrm{Ca}^{2+}$ from its source (channel or carrier) to its target (transport protein or enzyme). Evidence provided by in vitro $\mathrm{Ca}^{2+}$ flux experiments using an artificial phospholipid membrane is presented in support of the above proposed concept, and results from stochastic simulations of $\mathrm{Ca}^{2+}$ trajectories within nanojunctions are discussed to substantiate its possible requirements. Finally, we analyze recent literature on $\mathrm{Ca}^{2+}$ lipid interactions, which suggest that $2 \mathrm{D}$ interfacial $\mathrm{Ca}^{2+}$ diffusion may represent an important mechanism of signal transduction in other biological systems characterized by high phospholipid surface-to-aqueous volume ratios.
\end{abstract}

\section{Introduction}

All rapid functions in the body are selectively regulated by the universal biological messenger: ionic calcium $\left(\mathrm{Ca}^{2+}\right)$. For this single ionic messenger to harmoniously control such a great range of biological mechanisms, it is crucial that its signals are delivered with pinpoint precision and millisecond timing. The endoplasmic reticulum (ER) is the main organelle that orchestrates this essential spatiotemporal precision of $\mathrm{Ca}^{2+}$ signalling via numerous different close contact sites or nano-junctions (NJ) with the plasma membrane (PM), mitochondria, lysosomes and other organelles [1]. NJs have been defined as cytoplasmic sub-compartments where membranes of different organelles appose each other within the nano-metre scale. Typically the limiting membranes are separated by $20 \mathrm{~nm}$ or less and the specialized signalling function has been shown to fail at separation distances greater than $50 \mathrm{~nm}[2] ;$ [3]; [4]. The main function of the NJs in cells is to precisely localize the $\mathrm{Ca}^{2+}$ signals to specific $\mathrm{Ca}^{2+}$ sensors positioned within organellar membrane or PM domains, while bypassing the bulk cytoplasm. For example, refilling of the sarcoplasmic reticulum (SR) of vascular smooth muscle cells, in order to maintain asynchronous $\left[\mathrm{Ca}^{2+}\right]_{\mathrm{cyt}}$ oscillations, is achieved by coupling $\mathrm{Ca}^{2+}$ entry via $\mathrm{Ca}^{2+}$-influx-mode NCX (rNCX) to SERCA at PM-SR NJs, thus bypassing the bulk cytoplasm [5]. Employing stochastic particle simulator modelling software and using known NCX and SERCA turnover rates and surface densities, it was possible to generate a computational model of this cellular signalling process, which demonstrated that the rate of $\mathrm{Ca}^{2+}$ entry via rNCX/SERCA was indeed sufficient for replacing the $\mathrm{Ca}^{2+}$ released by periodic opening and closing of $\mathrm{IP}_{3} \mathrm{Rs}$ during the activation of asynchronous cytoplasmic $\mathrm{Ca}^{2+}$ waves that stimulate contraction [3]. However, to generate plausible predictions by our computational model, it was necessary to implement a $\mathrm{Ca}^{2+}$ target size on SERCA of approximately $20 \mathrm{~nm}^{2}$, which is 2500 
times larger than the area occupied by the dehydrated $\mathrm{Ca}^{2+}$ with a diameter of $1 \AA$. Assuming that short range local electrostatic forces of attraction between the fixed negatively charged binding sites on the SERCA macromolecule and the positively charged $\mathrm{Ca}^{2+}$ would increase the effective target size to an area several times larger than the size of the non-hydrated $\mathrm{Ca}^{2+}$, it would still be orders of magnitude smaller than 20 $\mathrm{nm}^{2}$. Therefore, in order to achieve effective functional transfer of $\mathrm{Ca}^{2+}$ from NCX on the PM to SERCA on the SR, it appears that an additional, as yet ignored, mechanism is operative to support the linkage between $\mathrm{Ca}^{2+}$ signalling elements (sources and sinks) within NJs, besides the proximity of the PM and SR in these regions. To this end, all available computational modelling data prompt us to conclude that, by itself, a 3D random walk of the $\mathrm{Ca}^{2+}$ between their sources and sinks on the two closely apposing membrane surfaces may be insufficient for efficient NJ $\mathrm{Ca}^{2+}$ signalling. We thus propose that, in addition, 2D exchange diffusion of $\mathrm{Ca}^{2+}$ on the targeted surface of the NJ has the potential to increase the efficiency of $\mathrm{Ca}^{2+}$ reaching its target.

\section{Background}

It is well established that $\mathrm{Ca}^{2+}$ can move rapidly through negatively charged solid lattices, such as fluorapatite, by the process of exchange diffusion [6]. An analogous model, featuring negatively charged phospholipids (PLs), has been described some time ago [7]; [8]; [9]. This experimental model consists of a millipore filter impregnated with a mixture of phospholipids of animal origin, separating two aqueous phases and exhibits properties that are highly relevant to the topic of $\mathrm{Ca}^{2+}$ movements through narrow aqueous passages lined by PLs. Its salient feature is that it supports net transfer of $\mathrm{Ca}^{2+}$ through relatively long PL lined pores at a much faster rate than would be possible for free diffusion within the limited adjoining aqueous phase. The mechanism that was presented to explain $\mathrm{Ca}^{2+}$ transport through this solid ion exchange membrane involves the association of $\mathrm{Ca}^{2+}$ with a negatively charged phosphate or carboxyl group of the PL surface on the cis-side of the membrane, followed by transfer of $\mathrm{Ca}^{2+}$ within a 2-dimensional matrix of similar sites, constituted by the pore-lining PL layers, and a final step of dissociation of $\mathrm{Ca}^{2+}$ from the negatively charged PL head groups on the trans-side of the membrane. The rate of this PL-mediated transport of radioactive labeled $\mathrm{Ca}^{2+}$ through the membrane was decreased by removal of $\mathrm{Ca}^{2+}$ from the buffered solution on the trans-side of the membrane. Paradoxically, further removal of remaining $\mathrm{Ca}^{2+}$ from the trans-solution by the addition of the soluble, but non-permeant chelator EDTA, increased the rate of PL-mediated net $\mathrm{Ca}^{2+}$ transport across the PL lined millipore filter by more than one order of magnitude. The mechanism proposed to explain the latter observation is that dissociation of $\mathrm{Ca}^{2+}$ from PL head groups on the trans-side of the membrane is rate-limiting and addition of a freely diffusible, impermeant $\mathrm{Ca}^{2+}$ binding site on EDTA, to the trans-side of the flux chamber facilitates the dissociation of PL bound $\mathrm{Ca}^{2+}$. Once the $\mathrm{Ca}^{2+}$ transported from the cis-side of the flux chamber has been chelated by EDTA, which is dissolved in a large volume of buffered solution on the trans-side, it cannot rebind to the PL membrane, but is replaced by the next

$\mathrm{Ca}^{2+}$ arriving from the cis-side. In this model system, electro-neutrality is preserved by the movement of $\mathrm{Mg}^{2+}$ and monovalent cations in the opposite direction. The mechanism envisioned for the transfer of $\mathrm{Ca}^{2+}$ from the PL membrane to EDTA involves an intermediary step of partial dissociation from the negative PL site and simultaneous association with a carboxyl group of EDTA. We propose herein, that a similar mechanism is involved in the transfer of $\mathrm{Ca}^{2+}$ bound to PL head-groups of the membranes lining the NJ to its biological target site on the $\mathrm{Ca}^{2+}$-receptor protein.

\section{Model Description}

When we compare the artificial PL-mediated $\mathrm{Ca}^{2+}$ transport in the model described above with biological PLlined nano-spaces some striking parallels become obvious. For example, rod outer segments of the bovine eye feature a 15-nm wide cytoplasmic phase between the intercalated discs, which stretch for several microns [10]. The lipid bilayers lining this narrow space contain $45 \%$ phosphatidylethanolamine, $36 \%$ phosphatidylcholine and $16 \%$ phosphatidylserine, calculated as percent of the total PL. At physiological $\mathrm{pH}$, phosphatidylcholine and phosphatidylethanolamine are zwitterions and phosphatidylserine has one net negative charge [10]. In 
this example, it was calculated that of the $\mathrm{Ca}^{2+}$ released during stimulation, $90 \%$ to $99 \%$ was bound to the PL-head groups of membranes Iining the nano-spaces between the intercalated discs, which is similar to the high ratio of bound/free $\mathrm{Ca}^{2+}$ in the above model membrane. Although historically it has been accepted that such binding slows diffusion, due to a drastic decrease in freely diffusible $\mathrm{Ca}^{2+}$ in the aqueous phase, it is also possible that the PL-bound $\mathrm{Ca}^{2+}$ within the water-PL membrane interface of the nano-space continues its trajectory by the mechanism of $2 \mathrm{D} \mathrm{Ca}^{2+}$ exchange diffusion. We therefore propose that the mechanism of $2 \mathrm{D} \mathrm{Ca}^{2+} / \mathrm{Mg}^{2+}, \mathrm{K}^{+}$exchange diffusion at the aqueous/phospholipid interfaces of NJs facilitates targeting of $\mathrm{Ca}^{2+}$ receptors located on organellar membranes and the inner PM.

Returning to the example of NCX-mediated SR refilling in vascular smooth muscle, we propose that $\mathrm{Ca}^{2+}$ enters the NJ via the reverse mode of NCX located in the junctional domain of the PM. It will then perform a 3D random walk and when it hits the negatively charged PL membrane boundary of the junctional nanospace proceeds along the lipid-water interface by a series of steps of reversible binding to negatively charged oxygen molecules for some variable time before being released back into the aqueous phase to resume its $3 \mathrm{D}$ random walk. After a number of such cycles the $\mathrm{Ca}^{2+}$ is envisioned to hit the ER membrane surface in the proximity of its target protein, in this case SERCA, which will then be reached more effectively by 2D surface exchange diffusion than would be expected if it depended solely on 3D diffusion in the aqueous phase. 


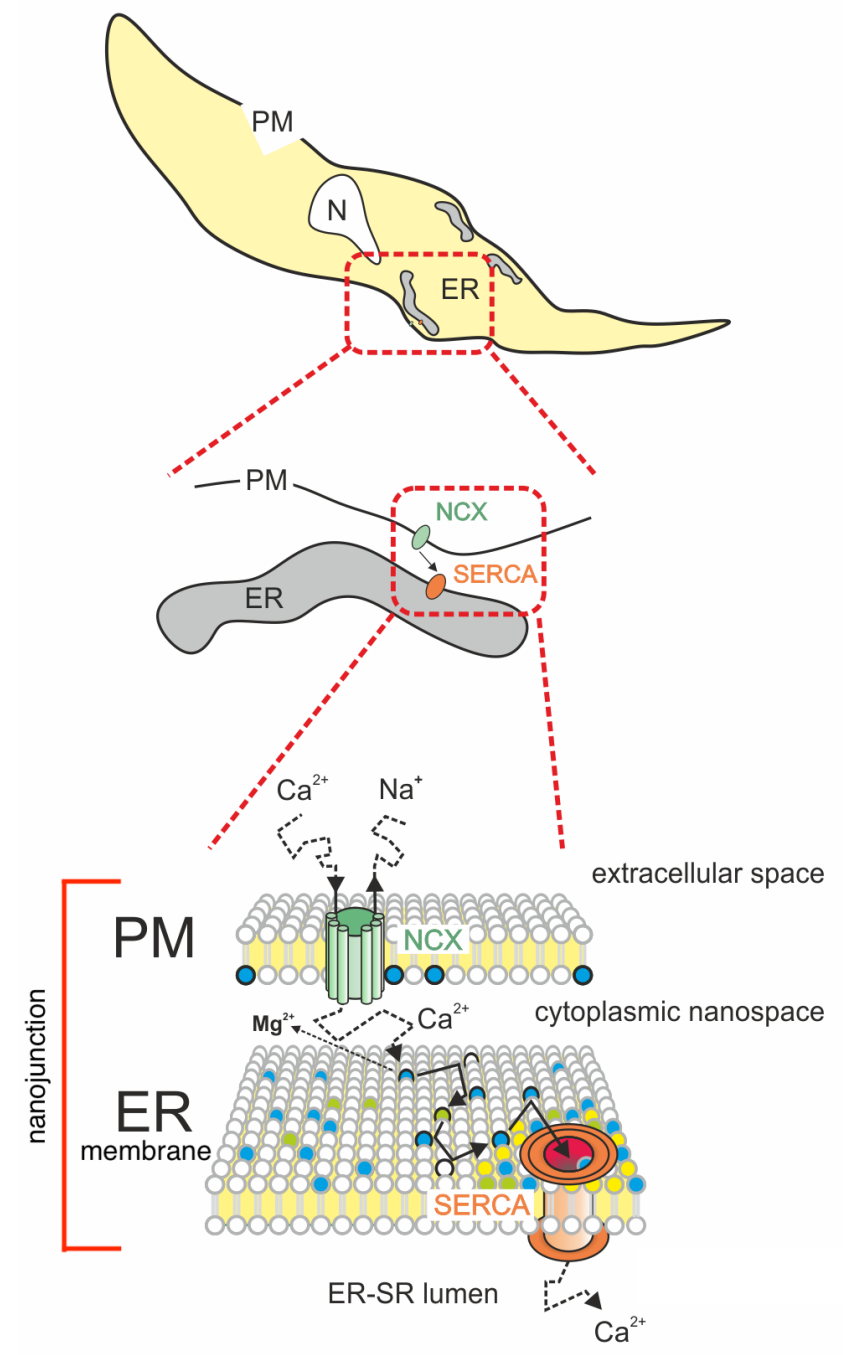

Figure 1: This cartoon illustrates a simplified hypothetical mechanism for refilling the ER at a nano-junction (NJ) between the PM and ER. The proposed process of $\mathrm{Ca}^{2+}$ transfer between extracellular space and ER lumen is depicted in a cut-out representing a distinct PM- and ER-membrane-delimited nanospace (lower panel) of a cell (upper panel). $\mathrm{Ca}^{2+}$ enters the NJ via rNCX, where it performs a 3D random walk and when it hits the ER surface it proceeds by $2 \mathrm{D}$ exchange diffusion on the negatively charged heterogeneous PL surface of the peripheral ER. During the $\mathrm{Ca}^{2+}$ pulse most of the $\mathrm{Mg}^{2+}$ associated with fixed coordination sites at the NJ membrane surface will be displaced into the aqueous phase. Both annular (yellow circles) and non-annular (non-yellow circles) lipids of the $\mathrm{Ca}^{2+}$ target protein may further facilitate guidance of $\mathrm{Ca}^{2+}$ into the transmembrane transport sites of SERCA.

A simplified model for this mechanism, which incorporates the $\mathrm{PL}, \mathrm{Ca}^{2+}$ surface exchange diffusion described for the artificial membrane [9] with the dynamic modelling of SR $\mathrm{Ca}^{2+}$ refilling [3], is illustrated in figure 1. This model predicts that 2D surface exchange diffusion will facilitate $\mathrm{Ca}^{2+}$ reaching its target in two ways: 1. by augmenting the rate of $\mathrm{Ca}^{2+}$ movement towards its target and thus enhancing the speed and intensity of the signal and 2. by decreasing the loss of $\mathrm{Ca}^{2+}$ at the NJ edges, further increasing the speed as well as the selectivity of $\mathrm{Ca}^{2+}$ signalling. 


\section{Discussion}

Due to the novelty of this concept, there are to date no experimental or computational tests reported with respect to the biological implications of $2 \mathrm{D} \mathrm{Ca}^{2+}$ exchange diffusion, specifically for its role in junctional $\mathrm{Ca}^{2+}$ signalling. On the other hand, non-Brownian interfacial diffusion has been intensively studied in physical chemistry, using state of the art techniques of single-molecule tracking and dynamic modelling [11]. A complex process of "Continuous Time Random Walk" (CTRW), which contains elements of "flying, hopping and crawling" and combining random walk 3D diffusion, adsorption and desorption as well as 2D surface diffusion, has been well documented. Elementary processes of intermittent, surface-delimited (2D) diffusion are considered important in molecular recognition and chemical sensing at interfacial surfaces. It is fascinating, that CTRW-type motion resembles trajectories of biological organisms executing foraging behaviour and could theoretically also be successfully applied to targeting of physiological receptors by signalling molecules [11]. With respect to biological $\mathrm{Ca}^{2+}$-signalling, it is particularly relevant that the ultra-structure of the endoplasmic reticulum as well as its appositions to other organelles exhibit features, such as tortuosity, binding affinity and high surface-to-aqueous volume ratios, a combination of which is prerequisite for the elementary processes of intermittent surface diffusion.

The physical chemistry of $\mathrm{Ca}^{2+}$ interactions with lipid bilayers has also been extensively studied and shows that $\mathrm{Ca}^{2+}$ binding can lower negative surface charge [8]; [12], cause clustering of PLs [12]; [13] and promote membrane fusion events as well as anchoring proteins in the lipid bilayer [14]. The structural effects of $\mathrm{Ca}^{2+}$ binding to PLs require millimolar concentrations of $\mathrm{Ca}^{2+}$, such as seen in the extracellular space and the ER lumen [15]. On the other hand, the cytoplasmic surfaces of cellular membranes are exposed to sub-micromolar $\mathrm{Ca}^{2+}$ concentrations, which are three orders of magnitude lower than the cytoplasmic concentration of $\mathrm{Mg}^{2+}$ and at least under resting conditions, will contain minimal bound $\mathrm{Ca}^{2+}$. Nonetheless, phosphate, carboxyl and carbonyl groups are effective $\mathrm{Ca}^{2+}$ binders at low concentrations and will buffer $\mathrm{Ca}^{2+}$ during transient elevations typically associated with $\mathrm{Ca}^{2+}$-signalling events. Recent studies in model membranes suggest that $\mathrm{Ca}^{2+}$-induced $\mathrm{PIP}_{2}$ clustering and $\mathrm{Ca}^{2+}$ coordination within a hydrogen-bond network formed by $\mathrm{PL}$ head groups occurs at membrane surfaces at micromolar divalent cation concentrations [16]. Hence, the generation of a dynamic cation-binding matrix at PL-cytoplasmic interfaces appears plausible for membrane regions endowed with a sufficient level of PLs and exposed to transient $\mathrm{Ca}^{2+}$ rises. In essence, the negatively charged cytoplasmic surface of NJs provides all the features required for $2 \mathrm{D}$ surface $\mathrm{Ca}^{2+}$-exchange-diffusion, which could promote efficient coupling between $\mathrm{Ca}^{2+}$ sources and $\mathrm{Ca}^{2+}$ targets during activation. A parallel mechanism of lateral diffusion of $\mathrm{Ca}^{2+}$ tightly bound to PLs in a monolayer can also not be excluded at this time, although the lateral mobility of free PLs in cell membranes is limited to a diffusivity of approximately $10 \mu \mathrm{m}^{2} / \mathrm{s}$, further slowed down in complexes with divalent cations [13] and presumably even less mobile in the vicinity of signalling proteins [17]. On the other hand, a matrix of PL head-groups, bridged by divalent cations, as suggested for PL-rich domains around $\mathrm{Ca}^{2+}$ signalling complexes in the context of PL signalling [18], may provide the ideal conditions for efficient linkage of $\mathrm{Ca}^{2+}$ sources to downstream targets via $2 \mathrm{D}$ surface $\mathrm{Ca}^{2+}$-exchange diffusion. Support in favour of such a membrane-delimited transfer of $\mathrm{Ca}^{2+}$ signals is derived from current concepts of tight interactions between membrane phospholipids and $\mathrm{Ca}^{2+}$ transport proteins and the well documented functional relevance of these interactions. In this context it is intriguing that PLs are a prominent component of the "annular lipid" shells, which typically surround transmembrane proteins involved in $\mathrm{Ca}^{2+}$ handling [19]; [20]; [21]. The role of these annular lipids for cation transport is still incompletely understood, but has been proposed to include the accumulation of cations in the vicinity of the transport molecule to promote transport efficiency and specificity [22]. Recent detailed insights into the structure of $\mathrm{Ca}^{2+}$ signalling complexes by crystallography and single particle CryoEM studies identified "non-annular" lipids, including PLs, which protrude into fenestrations and crevices of integral membrane proteins to govern $\mathrm{Ca}^{2+}$ transport and $\mathrm{Ca}^{2+}$ regulatory or sensory functions [23]; [24]; [25]; [26]. Of note, such non-annular, "structural" PLs were found to reside within clefts of the SERCA complex to determine the handling of $\mathrm{Ca}^{2+}$ within the transporter [19]. Interestingly, for SERCA, a distinct "path structure" was proposed to guide the $\mathrm{Ca}^{2+}$ ion from the membrane surface through hydrophilic clefts towards the central binding pocket [27]. It is tempting to speculate that this path, which is represented by 
a row of coordination sites (carbonyls) within the transmembrane protein complex, extends and connects to the surrounding annular cation-PL matrix, which supplies $\mathrm{Ca}^{2+}$ to the transporter via $2 \mathrm{D}$ exchange diffusion. Hence, we hypothesize that the unique architecture of NJs, along with the organization of membrane proteins within a specialized lipid environment, allows for exceptionally efficient and specific transfer of $\mathrm{Ca}^{2+}$ between sources and target sites. This proposed concept of $\mathrm{NJ} \mathrm{Ca}^{2+}$ transfer combines $3 \mathrm{D}$ random walk of $\mathrm{Ca}^{2+}$ within the junctional nanospace with a process of $2 \mathrm{D}$ interfacial surface diffusion that feeds $\mathrm{Ca}^{2+}$ into the acceptor and guidance machinery of target molecules.

\section{Conclusion}

We propose that $2 \mathrm{D} \mathrm{Ca}^{2+}$ exchange diffusion on negatively charged surfaces of biological phospholipids, as documented earlier in an inanimate model membrane [9], functions in guiding $\mathrm{Ca}^{2+}$ to its receptor sites embedded on biological membranes. Direct experimental or computational evidence for an involvement of this process of $\mathrm{NJ} \mathrm{Ca}^{2+}$ transfer is still awaited. Nonetheless, combining evidence from stochastic modelling and various computational as well as experimental studies with membrane models, it appears that 2D surface diffusion of $\mathrm{Ca}^{2+}$ at the interfaces of PL membranes and narrow aqueous phases of NJs, is both feasible and essential for specific targeting of calcium sensitive functional proteins embedded in the PL bilayers. Stochastic modelling of the targeting of $\mathrm{Ca}^{2+}$ receptors in NJs predicts that $\mathrm{Ca}^{2+}$ transfer from source to target cannot be accomplished on the basis of sole 3D random walk, but becomes plausible when involving 2D exchange-diffusion at the membrane surface, which accommodates the target. In addition, 2D interfacial $\mathrm{Ca}^{2+}$ diffusion may play an important role in signalling functions of other membrane systems characterized by high PL surface-to-aqueous volume ratios, such as diads and triads in cardiac and skeletal muscle [28], the narrow tubular segments of the ER involved in $\mathrm{Ca}^{2+}$ tunnelling [29], the narrow clefts in the nuclear envelope [30], the luminal surfaces of the nuclear envelope itself, as well as the cytoplasmic surfaces of ER containing RyRs and $\mathrm{IP}_{3}$ Rs generating cellular $\mathrm{Ca}^{2+}$ waves [31].

\section{References}

[1]C. van Breemen, N. Fameli, A.M. Evans, Pan-junctional sarcoplasmic reticulum in vascular smooth muscle: nanospace Ca2+ transport for site- and function-specific Ca2+ signalling., J Physiol. 591 (2013) 2043-54.

[2]C.H. Lee, K.H. Kuo, J. Dai, J.M. Leo, C.Y. Seow, C. van Breemen, Calyculin-A disrupts subplasmalemmal junction and recurring Ca2+ waves in vascular smooth muscle., Cell Calcium. 37 (2005) 9-16.

[3]N. Fameli, C. van Breemen, K.H. Kuo, A quantitative model for linking Na+/Ca2+ exchanger to SERCA during refilling of the sarcoplasmic reticulum to sustain $[\mathrm{Ca} 2+]$ oscillations in vascular smooth muscle., Cell Calcium. 42 (2007) 565-75.

[4]H.A.T. Pritchard, C.S. Griffin, E. Yamasaki, P. Thakore, C. Lane, A.S. Greenstein, S. Earley, Nanoscale coupling of junctophilin-2 and ryanodine receptors regulates vascular smooth muscle cell contractility., Proc Natl Acad Sci U S A. 116 (2019) 21874-21881.

[5]C.H. Lee, R. Rahimian, T. Szado, J. Sandhu, D. Poburko, T. Behra, L. Chan, C. van Breemen, Sequential opening of $\mathrm{IP}(3)$-sensitive $\mathrm{Ca}(2+)$ channels and SOC during alpha-adrenergic activation of rabbit vena cava., Am J Physiol Heart Circ Physiol. 282 (2002) H1768-77.

[6]E.E. Jay, P.M. Mallinson, S.K. Fong, B.L. Metcalfe, R.W. Grimes, Divalent cation diffusion in calcium fluorapatite, Journal of Materials Science. 46 (2011). https://doi.org/10.1007/s10853-011-5712-4.

[7]J.M. Tobias, D.P. Agin, R. Pawlowski, Phospholipidcholesterol membrane model. Control of resistance by ions or current flow., J Gen Physiol. 45 (1962) 989-1001.

[8]C. van Breemen, Permselectivity of a porous phospholipid-cholesterol artificial membrane. Calcium and lanthanum effects., Biochem Biophys Res Commun. 32 (1968) 977-83. 
[9]D. van Breemen, C. van Breemen, Calcium exchange diffusion in a porous phospholipid ion-exchange membrane., Nature. 223 (1969) 898-900.

[10]S. McLaughlin, J. Brown, Diffusion of calcium ions in retinal rods. A theoretical calculation., J Gen Physiol. 77 (1981) 475-87.

[11]D. Wang, D.K. Schwartz, Non-Brownian Interfacial Diffusion: Flying Hopping, and Crawling, The Journal of Physical Chemistry C. 124 (2020) 19880-19891. https://doi.org/10.1021/acs.jpcc.0c05834.

[12]K. Han, A. Gericke, R.W. Pastor, Characterization of Specific Ion Effects on PI(4,5) $\mathrm{P}_{\mathrm{i}} \mathrm{sub}_{\mathrm{i}} 2_{\mathrm{i}} / \mathrm{sub}_{\mathrm{i}}$ Clustering: Molecular Dynamics Simulations and Graph-Theoretic Analysis., J Phys Chem B. 124 (2020) $1183-1196$.

[13]R.P. Bradley, D.R. Slochower, P.A. Janmey, R. Radhakrishnan, Divalent cations bind to phosphoinositides to induce ion and isomer specific propensities for nano-cluster initiation in bilayer membranes, Royal Society Open Science. 7 (2020) 192208. https://doi.org/10.1098/rsos.192208.

[14]A. Melcrová, S. Pokorna, S. Pullanchery, M. Kohagen, P. Jurkiewicz, M. Hof, P. Jungwirth, P.S. Cremer, L. Cwiklik, The complex nature of calcium cation interactions with phospholipid bilayers., Sci Rep. 6 (2016) 38035 .

[15]M.J. Berridge, M.D. Bootman, H.L. Roderick, Calcium signalling: dynamics, homeostasis and remodelling., Nat Rev Mol Cell Biol. 4 (2003) 517-29.

[16]Y. Wen, V.M. Vogt, G.W. Feigenson, Multivalent Cation-Bridged PI(4,5)P2 Clusters Form at Very Low Concentrations, Biophysical Journal. 114 (2018) 2630-2639. https://doi.org/10.1016/j.bpj.2018.04. 048.

[17]T.K. Fujiwara, K. Iwasawa, Z. Kalay, T.A. Tsunoyama, Y. Watanabe, Y.M. Umemura, H. Murakoshi, K.G.N. Suzuki, Y.L. Nemoto, N. Morone, A. Kusumi, Confined diffusion of transmembrane proteins and lipids induced by the same actin meshwork lining the plasma membrane, Molecular Biology of the Cell. 27 (2016) 1101-1119. https://doi.org/10.1091/mbc.e15-04-0186.

[18]M.J. Sarmento, A. Coutinho, A. Fedorov, M. Prieto, F. Fernandes, Ca2+ induces PI(4,5)P2 clusters on lipid bilayers at physiological PI(4,5)P2 and Ca2+ concentrations, Biochimica Et Biophysica Acta (BBA) Biomembranes. 1838 (2014) 822-830. https://doi.org/10.1016/j.bbamem.2013.11.020.

[19]N.D. Drachmann, C. Olesen, J.V. Møller, Z. Guo, P. Nissen, M. Bublitz, Comparing crystal structures of Ca2+-ATPase in the presence of different lipids, FEBS Journal. 281 (2014) 4249-4262. https://doi. org/10.1111/febs.12957.

[20]Y. Gao, E. Cao, D. Julius, Y. Cheng, TRPV1 structures in nanodiscs reveal mechanisms of ligand and lipid action, Nature. 534 (2016) 347-351. https://doi.org/10.1038/nature17964.

[21]Conrard, Tyteca, Regulation of Membrane Calcium Transport Proteins by the Surrounding Lipid Environment, Biomolecules. 9 (2019) 513. https://doi.org/10.3390/biom9100513.

[22]J.A. Poveda, A.M. Giudici, M.L. Renart, M.L. Molina, E. Montoya, A. Fernández-Carvajal, G. Fernández-Ballester, J.A. Encinar, J.M. González-Ros, Lipid modulation of ion channels through specific binding sites, Biochimica Et Biophysica Acta (BBA) - Biomembranes. 1838 (2014) 1560-1567. https://doi.org/10.1016/j.bbamem.2013.10.023.

[23]M. Lichtenegger, O. Tiapko, B. Svobodova, T. Stockner, T.N. Glasnov, W. Schreibmayer, D. Platzer, G.G. de la Cruz, S. Krenn, R. Schober, N. Shrestha, R. Schindl, C. Romanin, K. Groschner, An optically controlled probe identifies lipid-gating fenestrations within the TRPC3 channel, Nature Chemical Biology. 14 (2018) 396-404. https://doi.org/10.1038/s41589-018-0015-6.

[24]R. Cai, X. Liu, R. Zhang, L. Hofmann, W. Zheng, M.R. Amin, L. Wang, Q. Hu, J.-B. Peng, M. Michalak, V. Flockerzi, D.W. Ali, X.-Z. Chen, J. Tang, Autoinhibition of TRPV6 Channel and Regulation by PIP2, 
IScience. 23 (2020) 101444. https://doi.org/10.1016/j.isci.2020.101444.

[25]D. Barth, A. Lückhoff, F.J.P. Kühn, Species-Specific Regulation of TRPM2 by PI(4,5)P2 via the Membrane Interfacial Cavity, International Journal of Molecular Sciences. 22 (2021) 4637. https: //doi.org/10.3390/ijms22094637.

[26]L.M. Espinoza-Fonseca, Probing the effects of nonannular lipid binding on the stability of the calcium pump SERCA, Scientific Reports. 9 (2019). https://doi.org/10.1038/s41598-019-40004-y.

[27]C. Toyoshima, M. Nakasako, H. Nomura, H. Ogawa, Crystal structure of the calcium pump of sarcoplasmic reticulum at 2.6 A resolution., Nature. 405 (2000) 647-55.

[28]C. Franzini-Armstrong, The relationship between form and function throughout the history of excitationcontraction coupling., J Gen Physiol. 150 (2018) 189-210.

[29]O.H. Petersen, R. Courjaret, K. Machaca, Cajsup $2+i / \sup i$ tunnelling through the ER lumen as a mechanism for delivering $\mathrm{Ca}_{j} \mathrm{sup}_{i} 2+i / \sup _{i}$ entering via store-operated Cajsup $i 2+i /$ sup $_{i}$ channels to specific target sites., J Physiol. 595 (2017) 2999-3014.

[30]J. Duan, J. Navarro-Dorado, J.H. Clark, N.P. Kinnear, P. Meinke, E.C. Schirmer, A.M. Evans, The cellwide web coordinates cellular processes by directing site-specific Cajsup $i 2+i / s_{i}$ flux across cytoplasmic nanocourses., Nat Commun. 10 (2019) 2299.

[31]M. Iino, H. Kasai, T. Yamazawa, Visualization of neural control of intracellular Ca2+ concentration in single vascular smooth muscle cells in situ., EMBO J. 13 (1994) 5026-31. 